$\underline{\text { Research article }}$

\title{
Asymptomatic Bacteriuria in Pregnancy: Prevalence, Risk factors and Causative Organisms
}

\author{
Perera Jennifer, ${ }^{1}$ Randeniya Cyril,${ }^{2}$ Perera Piyumi, ${ }^{1}$ Gamhewage Nimesha, ${ }^{1}$ Jayalathaarachchi \\ Renuka $^{1}$ \\ Sri Lankan Journal of Infectious Diseases 2012 Vol.1(2); 42-46 \\ DOI: http://dx.doi.org/10.4038/sljid.v2i1.3810
}

Key words: Asymptomatic bacteriuria; pregnancy; prevalence; risk factors

\begin{abstract}
Untreated bacteriuria during pregnancy is associated with adverse maternal and perinatal outcomes. It is cost effective to screen for bacteriuria if the prevalence rate is $2 \%$ or more. The prevalence rate in this study was 3.6\%. $67 \%$ of the aetiological agents belonged to the coliform group and balance 33\% were staphylococci. All isolates were sensitive to nitrofurantoin. There was no association between bacteriuria and risk factors such as gestational diabetes, past urinary tract infection, multiparity, advanced maternal age, lower education level, advanced gestational age and lower socioeconomic status. Screening of pregnant mothers is reported to be cost effective if the prevalence rates are $\geq 2 \%$. It is recommended to screen pregnant mothers early in their pregnancy and treat those with significant bacteriuria as this could significantly minimize adverse maternal and foetal outcomes.
\end{abstract}

\section{Introduction}

Urinary tract infection (UTI) during pregnancy could be either symptomatic or asymptomatic. A UTI may manifest as an asymptomatic bacteriuria, acute cystitis or pyelonephritis. Untreated bacteriuria during pregnancy is associated with adverse maternal and perinatal outcomes. Globally asymptomatic bacteriuria affects $2-10 \%$ of all pregnant women. ${ }^{1}$ Adverse maternal outcomes include symptomatic cystitis and development of pyelonephritis (in up to 30\%) and

${ }^{1}$ Department of Microbiology, Faculty of Medicine, Colombo, Sri Lanka

${ }^{2}$ Department of Obstetrics and Gynaecology, Faculty of Medicine, Colombo, Sri Lanka

Address for correspondence: Jennifer Perera, Senior Professor of Microbiology, Department of Microbiology, Faculty of Medicine, P O Box 271, University of Colombo, Sri Lanka .

Email address: jennifer_perera55@yahoo.com 
preterm labour and delivery. Pyelonephritis in the pregnant patient leads to septicaemia in 10 $20 \%$ of cases and acute respiratory distress syndrome in $2 \%$. The associated adverse foetal outcomes include prematurity, low birth weight and increased perinatal mortality ${ }^{2}$.

In addition there are increased maternal risks reported for pre-eclampsia, anaemia, chorioamnionitis and post-partum endometritis in patients with significant bacteriuria. Fetal risks include fetal growth retardation, stillbirth, mental retardation and development delay. It is postulated that direct bacterial endotoxin damage is responsible.

Under normal circumstances, the relatively acidic $\mathrm{pH}$, high osmolality and high urea concentration in urine is generally bacteriostatic to most bacteria. Furthermore, in an anatomically normal urinary tract, infection is prevented by unobstructed antegrade flow of urine. The physiological changes, both hormonal and mechanical that occur in the urogenital tract during pregnancy, increases the potential for colonization by pathogenic bacteria. During pregnancy the bladder volume increases and detrusor tone decreases. Additionally, progesterone relaxes ureteric smooth muscle causing dilatation of ureters, which is further aggravated due to pressure from the expanding uterus. All these factors lead to urinary stasis, dysfunctional ureteric valves and vesicoureteric reflux, which facilitates bacterial colonization and ascending infection. Seventy percent of pregnant women develop glycosuria and this in combination with the physiological lowering of urine osmolality will further facilitate bacterial colonization ${ }^{3}$.

It has been demonstrated through randomized trials that antimicrobial treatment of asymptomatic bacteriuria during pregnancy will decrease the risk of subsequent pyelonephritis from $20-35 \%$ to $1-4 \%$ and the risk of having a low birth weight baby from $15 \%$ to $5 \%{ }^{4}$. The antenatal urine culture routine for all pregnant women is costly and not feasible in many parts of the developing world. Selection of mothers for screening with risk factors may reduce the necessity of urine culture for all pregnant women and may be used as an appropriate alternative strategy of management. Gestational diabetes, past urinary tract infection, multiparity, advanced maternal age, lower education level, advanced gestational age and lower socioeconomic status have been reported as risk factors in some of the studies, and conflicting results have been obtained from different studies. ${ }^{5,6}$

Quantitative culture is the gold standard for diagnosis of bacteriuria. However a pregnant woman with a positive dipslide test is very likely to have a definitive diagnosis of asymptomatic bacteriuria, whereas a negative result effectively rules out the presence of bacteriuria. When tested with dipsticks, only $2 \%$ of pregnant women with asymptomatic bacteriuria will not receive antibiotic treatment when necessary and fewer than $1 \%$ of pregnant women without asymptomatic bacteriuria would be classified as positive. ${ }^{7}$ However, dipsticks that measure nitrites and leukocyte esterase have low sensitivity for use in screening for asymptomatic bacteriuria during pregnancy. $^{7}$

No information is available from Sri Lanka on the prevalence of asymptomatic bacteriuria in pregnant women. Hence this prospective study was conducted to determine the prevalence of asymptomatic bacteriuria, risk factors, their degree of association, and aetiological agents in a selected group of Sri Lankan pregnant women. 


\section{Materials and Methods}

A descriptive cross sectional study was conducted on 250 pregnant women attending the University Unit antenatal clinic of the De Soysa Maternity Hospital. They were recruited to the study during their first clinic visit irrespective of their age, race or parity. A pre tested, selfadministered questionnaire was used to collect the socio demographic data and data on possible risk factors. They were screened for asymptomatic bacteriuria using quantitative culture of a sample of midstream urine within two hours of collection. The specific instructions for collection of midstream urine specimens were provided on site by two of the authors to enable patients to collect appropriate specimens. The urine samples were cultured on CLED medium using a calibrated wire loop. A colony count of more than $10^{5}$ per $\mathrm{ml}$ of urine was taken as significant bacteriuria. Data were analyzed using Statistical Package for Social Sciences (SPSS) version17.0. The ethical clearance for the study was obtained from the Ethics Review Committee of the Faculty of Medicine, Colombo.

\section{Results}

Among the 250 women screened for asymptomatic bacteriuria, 9 showed a significant colony count resulting in prevalence rate of $3.6 \%$. There was no significant association between bacteriuria and the risk factors previously described; gestational diabetes, past urinary tract infection, multiparity, advanced maternal age, lower education level, advanced gestational age and lower socioeconomic status when analyzed using the Fisher exact test. The causative bacteria were coliforms $(\mathrm{n}=6)$, Staphylococcus aureus $(\mathrm{n}=2)$ and Staphylococcus saprophyticus $(n=1)$. All were sensitive to the first line antimicrobials, norfloxacin and nitrofurantoin. Four of the nine isolates were resistant to ampicillin. In addition, two of the coliforms were resistant to cephalexin and cefuroxime.

\section{Discussion}

A cost evaluation study reported that screening for pyelonephritis is appropriate when the prevalence of asymptomatic bacteriuria is greater than $2 \%$. In this study we saw a prevalence of $3.6 \%$ and screening of all antenatal women for asymptomatic bacteriuria by a quantitative urine culture is recommended. ${ }^{8}$

Coliforms were the most common pathogens (67\%) associated with asymptomatic bacteriuria in our study, and they represented at least $80 \%$ of isolates in previous studies ${ }^{9}$. Among coliforms Escherichia coli is reported to be the commonest. The small number of positive women in this study did not provide a significant link between the previously described risk factors and the development of asymptomatic bacteriuria and this may require investigation using a larger cohort of women selectively drawn from different risk groups. As this is the first study that describes the prevalence of asymptomatic bacteriuria in pregnant women, further studies to determine risk factors for Sri Lankan women may be deemed necessary as risk factors varied vastly between different geographical settings. $5,6,9,11$ 
Treatment of asymptomatic bacteriuria has been shown to reduce the rate of pyelonephritis in pregnancy and therefore screening for and treatment of asymptomatic bacteriuria has become a standard of obstetrical care. ${ }^{10}$ Oral nitrofurantoin is a good antibiotic choice for treatment of pregnant women with asymptomatic bacteriuria and all strains isolated in this preliminary study showed sensitivity to nitrofurantoin. Although ampicillin and oral cephalosporins are useful and safe alternatives with a lower incidence of adverse effects, a significant number of isolates showed resistance to these antibiotics. Thus, the antibiotic sensitivity patterns should be used in determining therapy as inappropriate therapy has been responsible for recurrences of asymptomatic bacteriuria with development of acute pyelonephritis later. ${ }^{11}$ Although all strains were susceptible to norfloxacin, it is not recommended when safer alternatives are available for use during pregnancy. Women with acute pyelonephritis may sustain significant complications, such as preterm labor, transient renal failure, ARDS, sepsis and shock, and haematologic abnormalities. Therefore it is important to screen all pregnant women for the presence of bacteriuria at their first prenatal visit, preferably in the first trimester and those who are positive should be followed up closely after treatment because as many as one third will experience a recurrence. ${ }^{12}$ A 'test of cure' urine culture 2 weeks after completion of treatment is required to ensure response to therapy. If the 'test of cure' culture is positive a repeat course of antibiotics is recommended based on urine culture and sensitivity report and suppressive therapy (e.g. Nitrofurantoin $50 \mathrm{mg} /$ day) is recommended after a negative 'test of cure' urine culture. ${ }^{13}$ It is also recommended that patients with two or more episodes of bacteriuria are followed up with monthly repeat cultures until delivery to ensure urine sterility during the pregnancy. ${ }^{13}$

\section{References}

1. Delzell JE Jr., \& Lefevre ML. Urinary tract infections during pregnancy. American Family Physician 2000; 61 (3) 713- 721. No doi.

2. Mittendorf R, Williams MA, Kass EH. Prevention of preterm delivery and low birth weight associated with asymptomatic bacteriuria. Clinical Infectious Disease 1992; 14: 927-32. No doi.

3. Asscher AW, Sussman M, Waters WE, Davis RH, Chick S. Urine as a medium for bacterial growth. The Lancet 1966; 2: 1037-41. No doi.

4. Smaill F, Vazquez JC. Antibiotics for asymptomatic bacteriuria in pregnancy. Cochrane Database of Systematic Reviews 2007, Issue 2. Art. No.: CD000490. doi: 10.1002/14651858.CD000490.pub2.

5. Akinloye O, Ogbolu DO, Akinloye OM, Terry Alli OA. Asymptomatic bacteriuria of pregnancy in Ibadan, Nigeria: a re-assessment. Br J Biomed Sci 2006; 63: 109-12. No doi.

6. Fatima N, Ishrat S. Frequency and risk factors of asymptomatic bacteriuria during pregnancy. J Coll Physicians Surg Pak 2006; 16: 273-5. No doi.

7. Mignini L, Carroli G, Abalos E, Widmer M, Amigot S, Nardin JM, et al; World Health Organization Asymptomatic Bacteriuria Trial Group. Accuracy of diagnostic tests to detect asymptomatic bacteriuria during pregnancy. Obstet Gynecol. 2009 Feb;113(2 Pt 1):346-52. No doi.

8. Rouse DJ, Andrews WW, Goldenberg RL, Owen J. Screening and treatment of asymptomatic bacteriuria of pregnancy to prevent pyelonephritis: a cost-effectiveness and cost-beneficial analysis. Obstetrics \& Gynecology 1995;86:119-23. No doi. 
9. Eyal Sheiner, Efrat Mazor-Drey \& Amalia Levy. Asymptomatic bacteriuria during pregnancy. The Journal of Maternal-Fetal and Neonatal Medicine, 2009; 22(5): 423427. doi:10.1080/14767050802360783

10. Schnarra J \& Smaill J. Asymptomatic bacteriuria and symptomatic urinary tract infections in pregnancy . European Journal of Clinical Investigations 2008: 38 (S3) : 50 -7 . No doi.

11. Gilstrap LC \& Ramin SM. Urinary tract infections during pregnancy. Obstetrics and Gynaecology Clinics of North America 2001: 28(3) : 581-91. No doi.

12. Mackejko A M \& Schaeefer A J. Asymptomatic bacteriuria and symptomatic urinary tract infections during pregnancy. Urological Clinics of North America 2007; 34(1) : 35 -47. No doi

13. Chen KT. UTI in pregnancy: 6 questions to guide therapy. OBG Management 2004; 16(11): 36-54. No doi

\section{Acknowledgements}

This study was funded by the GlaxoSmithKline Pharmaceuticals, Sri Lanka 